\title{
A Drosophila DEG/ENaC Subunit Functions Specifically in Gustatory Neurons Required for Male Courtship Behavior
}

\author{
Elena Starostina, ${ }^{1,2}$ Tong Liu, ${ }^{1,2}$ Vinoy Vijayan, ${ }^{1,2}$ Zheng Zheng, ${ }^{3}$ Kathleen K. Siwicki, ${ }^{3}$ and Claudio W. Pikielny ${ }^{1,2}$ \\ ${ }^{1}$ Department of Genetics and ${ }^{2}$ Neuroscience Center, Dartmouth Medical School, Hanover, New Hampshire 03755-1404 and ${ }^{3}$ Department of Biology, \\ Swarthmore College, Swarthmore, Pennsylvania 19081
}

Detection of specific female pheromones stimulates courtship behavior in Drosophila melanogaster males, but the chemosensory molecules, cells, and mechanisms involved remain poorly understood. Here we show that $p p k 25$, a DEG/ENaC ion channel subunit required for normal male response to females, is expressed at highest levels in a single sexually dimorphic gustatory neuron of most taste hairs on legs and wings, but not in neurons that detect courtship-inhibiting pheromones or food. Synaptic inactivation of $p p k 25$-expressing neurons, or knockdown of $p p k 25$ expression in all gustatory neurons, significantly impairs male response to females, whereas gustatory expression of $p p k 25$ rescues the courtship behavior of $p p k 25$ mutant males. Remarkably, the only other detectable albeit significantly weaker expression of $p p k 25$ occurs in olfactory neurons implicated in modulation of courtship behavior. However, expression of $p p k 25$ in olfactory neurons is not required for male courtship under our experimental conditions. These data show that $p p k 25$ functions specifically in peripheral taste neurons involved in activation of courtship behavior, an unexpected function for this type of channel. Furthermore, our work identifies a small subset of gustatory neurons with an essential role in activation of male courtship behavior, most likely in response to female pheromones.

\section{Introduction}

In Drosophila melanogaster, initiation and modulation of the intricate courtship steps that precede mating involve complex communication between males and females (Villella and Hall, 2008; Dahanukar and Ray, 2011). While olfactory and gustatory receptors, sensory neurons, and even some of the central circuitry involved in detection and response to courtship-inhibiting pheromones have been identified (Kurtovic et al., 2007; Lacaille et al., 2007; van der Goes van Naters and Carlson, 2007; Datta et al., 2008; Laughlin et al., 2008; Miyamoto and Amrein, 2008; Yew et al., 2009; Koganezawa et al., 2010; Ronderos and Smith, 2010; Ruta et al., 2010; Wang et al., 2011), the cells and molecules involved in detecting pheromones that activate rather than inhibit courtship behavior are less understood, at least in part due to the involvement of several sensory modalities (Ejima and Griffith, 2008; Griffith and Ejima, 2009; Krstic et al., 2009). Both vision and hearing stimulate courtship initiation, but are not

Received Dec. 13, 2011; revised Jan. 30, 2012; accepted Feb. 16, 2012.

Author contributions: E.S., T.L., V.V., Z.Z., K.K.S., and C.W.P. designed research; E.S., T.L., V.V., and Z.Z. performed research; E.S., T.L., V.V., Z.Z., K.K.S., and C.W.P. analyzed data; C.W.P. wrote the paper.

This work was supported by Grant R01DC007911 from the NIDCD to C.W.P., and through an award from the Biomedical Research Support Program for Medical Schools from the Howard Hughes Medical Institute to Dartmouth Medical School (76200-560801).We are grateful to Michael Rosbash, Larry Zwiebel, Stephen Goodwin, Fred Marion-Poll, and Yashi Ahmed for suggestions on the manuscript. We thank the Bloomington Stock Center (Indiana University), the Transgenic RNAi project at Harvard Medical School, and the Vienna Drosophila RNAi Center for fly lines, and the Developmental Studies Hybridoma bank for a monoclonal antibody used in this study.

T.L. and V.V. contributed equally to this work.

Correspondence should be addressed to Claudio W. Pikielny, Department of Genetics and Neuroscience Center, Dartmouth Medical School, 3 Rope Ferry Road, Hanover, NH 03755-1404. E-mail: CWP@Dartmouth.edu.

DOI:10.1523/JNEUROSCI.6178-11.2012

Copyright $\odot 2012$ the authors $\quad 0270-6474 / 12 / 324665-10 \$ 15.00 / 0$ essential, while pheromone detection plays a central role. Olfactory neurons expressing the olfactory receptor Or $47 b$ detect unknown fly odors and contribute to a male's ability to find a female and initiate courtship (van der Goes van Naters and Carlson, 2007; Root et al., 2008; Wang et al., 2011). However, loss of olfaction by itself has only a modest effect on overall levels of courtship (Stockinger et al., 2005; Krstic et al., 2009). In contrast, mutants lacking functional taste hairs display significant reductions in courtship, and males lacking both olfactory and gustatory inputs display almost no response to females (Boll and Noll, 2002; Krstic et al., 2009). In addition, since the only characterized pheromones that activate male courtship are long chain unsaturated cuticular hydrocarbons with very low volatility, their detection may require direct contact with gustatory organs (Ferveur et al., 1997; Ferveur, 2005; Siwicki et al., 2005; Wicker-Thomas, 2007). Two members of the Gr family of gustatory receptors are important for male response to females; however, the cells in which these two genes function and the sensory modality involved are either unknown or controversial (Bray and Amrein, 2003; Ejima and Griffith, 2008; Watanabe et al., 2011). Therefore, while contact perception of female pheromones is likely critical to activation of male courtship behavior, the gustatory neurons involved have not been identified.

We previously reported that mutations inactivating $p p k 25$, a DEG/ENaC sodium channel subunit, impair activation of male courtship behavior in the presence of a female, but have no effect on behaviors unrelated to courtship, including walking, preening, and gustatory response to sugars and salts (Lin et al., 2005). Here we identify a small subset of gustatory neurons on the legs and wings that are specifically required for normal male courtship of females, and may therefore detect courtship-stimulating 
female pheromones. We further show that these gustatory neurons represent the site of $p p k 25$ 's function in activation of courtship behavior. These studies reveal that in addition to DEG/ENaCs that function in gustatory perception of sodium ions (Schiffman et al., 1983; Heck et al., 1984; Chandrashekar et al., 2010) or water molecules (Cameron et al., 2010; Chen et al., 2010); ppk25 plays an unexpected role in gustatory activation of courtship behavior.

\section{Materials and Methods}

Generation of ppk25-Gal4, Western blots, immunostaining, and RNA-ligase-mediated rapid amplification of cDNA ends. The ppk25-Gal4 construct was generated starting from a derivative of the $\mathrm{pP}\{\mathrm{CaSpeR}-4\}$ (Qian et al., 1991) vector containing additional rare restriction enzyme cutting sites (our unpublished data). The insert contains four distinct fragments in the following order (Fig. 1B): (1) a $5 \mathrm{~kb}$ fragment with sequences upstream of the $p p k 25$ start codon and flanked by synthetic AscI and PmeI sites; (2) the yeast Gal4 gene (Brand and Dormand, 1995) flanked by PmeI sites; (3) a 3832 bp PmeI-NotI fragment containing the entire $p p k 25$ coding region, inactivated by mutation of the ATG start codon to AAG, and $\sim 2 \mathrm{~kb}$ of sequences downstream of the ppk25 stop codon; and (4) a NotI-BamHI fragment of the pGATB vector containing the SV40 polyadenylation sequences (Brand and Dormand, 1995). Transgenic lines were generated using standard procedures (Rubin and Spradling, 1982). Immunostaining of antennae and brains was performed as described previously (Laissue et al., 1999) using rabbit anti-GFP (Invitrogen) at a dilution of 1:200, and anti-nc82 (Developmental Studies Hybridoma Bank, supported by the NICHD at the University of Iowa) at a dilution of 1:40. Western blots were performed with the same rabbit anti-GFP, and mouse anti- $\alpha$ tubulin (Sigma-Aldrich), at 1:500 and 1:1000 dilutions, respectively. For RNA analysis, body fractions (200 male front legs or 100 heads, without antennae) were dissected by hand, the RNA extracted as published (Starostina et al., 2009), and RNA-ligase-mediated rapid amplification of cDNA ends (RLM-RACE) performed using a kit from Ambion.

Behavioral assays. Flies were raised at $25^{\circ} \mathrm{C}, 50 \%$ relative humidity in a $12 \mathrm{~h}: 12 \mathrm{~h}$ light:dark cycle, except where indicated. For courtship assays, males were collected soon after eclosion and aged individually for 5-7 d before testing. Fifteen to twenty virgin wild-type females (either Canton-S or $w^{1118}$ ) were aged together for 5-7 d, then anesthetized and decapitated. After a short recovery, decapitated females that stood upright and preened were placed in the presence of a test male, and behavior was recorded with a digital Mini-DV camera for $10 \mathrm{~min}$. Except where indicated, observation was performed under infrared light in Plexiglas chambers $(7 \mathrm{~mm}$ diameter $\times 7 \mathrm{~mm}$ deep $)$. Behaviors were scored blind and analyzed using the LIFESONG X software (version 0.8) (Villella et al., 2005). The Courtship Index (CI) is the fraction of time the male spends performing any courtship behavior $\times 100$ (Hall, 1978), while we defined a Total Behavioral Index (TBI) as the fraction of the same $10 \mathrm{~min}$ period during which the male courts, walks, or preens $\times 100$. We find that the TBI provides a more objective measure of overall male activity than more specific parameters, e.g., measures of locomotor behavior, since reductions in courtship typically result in increased time spent performing behaviors unrelated to courtship but have little effect on the total behavior displayed. For CI and TBI, error bars indicating a 95\% confidence interval were calculated as the SEM, and the Wilcoxon rank sum test was used to determine statistical significance. For the fraction of males initiating courtship, error bars indicate $95 \%$ confidence intervals for binomial distributions, and statistical significance was calculated using a $\chi^{2}$ test for equality of distributions.
Gustatory response to sucrose was assayed by measuring the proboscis extension reflex (PER), as described previously (Heimbeck et al., 2001). Briefly, 5- to 7-d-old males were starved for $24 \mathrm{~h}$ in vials without food but containing water-soaked cotton wool. After $24 \mathrm{~h}$, males were anesthetized with $\mathrm{CO}_{2}$, attached to glass slides on their backs using myristic acid (Acros Organics), and allowed to recover for $2 \mathrm{~h}$ in saturating humidity. Each live fly was then given water until it no longer exhibited a PER when touched on its forelegs with a drop of water, at which point water was replaced with a $100 \mathrm{~mm}$ sucrose solution and the response tested five times with a delay of $30 \mathrm{~s}$ between tests to avoid habituation (Heimbeck et al., 2001). The PER index represents the number of times the male extended its proboscis in response to sucrose divided by 5 .

Drosophila stocks. Most fly lines used were obtained from the Bloomington Stock Center. Two RNAi lines were obtained from the Vienna Drosophila RNAi Center (stock numbers v101808 and v41512, targeting ppk25 and CG13895, respectively), and one RNAi line from the Transgenic RNAi Project at Harvard Medical School targeting ppk25 (stock number JF02434). The Poxn-Gal4, fru-LexA, and lexAop-FRT-tdTomato::nls; UAS-stinger lines were gifts from David Mellert.

\section{Results}

\section{A ppk25-Gal4 transgene reflects expression of the} endogenous ppk25 gene

To define the expression and function of ppk25 using the UAS/ Gal4 system (Elliott and Brand, 2008), we generated a transgenic construct in which the yeast transcriptional activator Gal4 is placed under control of $p p k 25$ regulatory regions. For many Drosophila genes, $3 \mathrm{~kb}$ of upstream sequences are sufficient to direct reporter gene expression in the pattern of the endogenous gene (Pfeiffer et al., 2008). In the case of ppk25, however, only $107 \mathrm{nt}$ separate the 3 ' end of the CheB42a mRNA from the ATG initiator codon for the Ppk25 protein (Lin et al., 2005; Ben-Shahar et al., 2007) (Fig. 1A). Given this unusually short intergenic region, sequences required for correct regulation of $p p k 25$ expression may occur upstream of the CheB42a gene or within it, or within the ppk25 gene itself, perhaps in an intron or in its $3^{\prime}$ untranslated region. To best reproduce the context of the original $p p k 25$ gene, we therefore inserted the Gal4 open reading frame immediately after the $p p k 25$ start codon, within a large genomic fragment that 


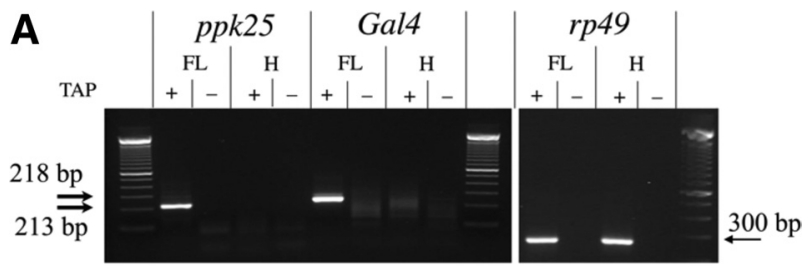

B

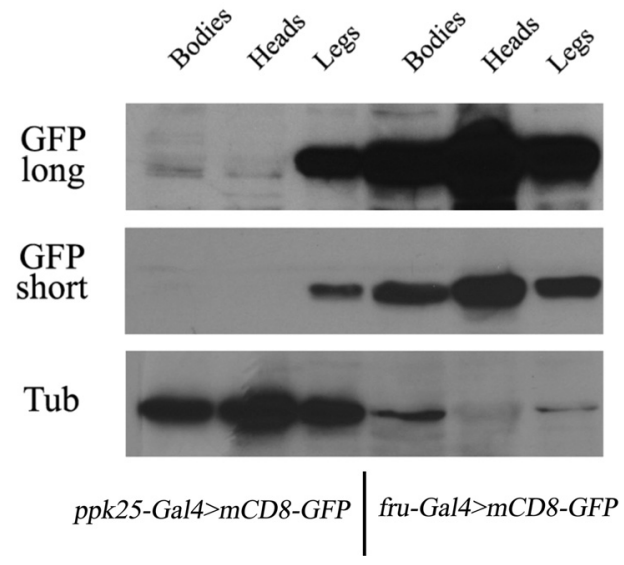

Figure 2. Both $p p k 25-G a / 4$ and the endogenous $p p k 25$ gene are expressed in legs but not heads.A, Transcripts of the ppk25-Gal4 transgene and of the endogenous ppk25 gene initiate at the same nucleotide, and are found in front legs but not in heads. RNA extracted from front legs (FL) or heads without antennae (H) was analyzed by amplification of CDNAs using RLM RACE, which only amplifies CDNAs produced by reverse transcription of $5^{\prime}$-capped RNAs (Schaefer, 1995). + and - indicate whether the RNA sample was treated with tobacco acid pyrophosphatase (TAP) to generate a 5 ' monophosphate required for ligation to the RNA adaptor. Specific antisense primers were used for ppk25, Gal4, and rp49. The unique bands of 218 and $213 \mathrm{bp}$ observed in CDNAs from front leg and resulting from amplification of the $p p k 25$ and Gal 4 transcripts, respectively, were sequenced, confirming the reported start site for the ppk25 mRNA (Ben-Shahar et al., 2007), and an identical start site for the ppk25-Gal4 transgene (Fig. 1). In contrast, the ubiquitous $r p 49 \mathrm{mRNA}$ yields a predicted band of $300 \mathrm{bp}$ that is detected equally by RLM-RACE using CDNA from front legs and heads. $\boldsymbol{B}$, Expression of GFP under control of $p p k 25$ $\mathrm{Gal} 4$ is detectable in legs but not heads or bodies. Extracts were made from bodies (without legs, wings, or heads), heads (without antennae), or legs of males expressing mCD8-GFP under control of either ppk25-Gal4 or fru-Gal4 (Stockinger et al., 2005) as indicated, and analyzed by immunoblotting with anti-GFP. The blots were then stripped and reprobed with anti-tubulin antibody (Tub), showing that for males containing the ppk25-Gal4 driver, more extract from heads than legs was loaded on the gel. For those males, extracts from heads and bodies show no detectable GFP band above the background of nonspecific bands. GFP short and GFP Iong refer to different exposures of the same Western blot.

contains all $p p k 25$ regulatory sequences required to rescue $p p k 25$ function in a deletion mutant (Lin et al., 2005). In this construct, the Gal4 gene is preceded by $5 \mathrm{~kb}$ of sequences normally found upstream of $p p k 25$, including the entire $C h e B 42 a$ gene and all sequences required for its regulation (Xu et al., 2002; Park et al., 2006), and is followed by the entire ppk25 coding region, inactivated by a point mutation in the initiator codon, and $\sim 2 \mathrm{~kb}$ of noncoding downstream sequences (Fig. $1 B$ ).

To test whether expression of ppk25-Gal4 accurately reflects that of the endogenous ppk25 gene, we compared the sites of transcription initiation and the tissue distribution of the two mRNAs using RLM-RACE, a method that only amplifies cDNAs resulting from reverse transcription of $5^{\prime}$-capped mRNAs (Schaefer, 1995). Specific primers allow amplification of the $5^{\prime}$ end, either of the endogenous ppk25 transcripts, or of transcripts from the ppk25-Gal4 transgene, yielding a single detectable RACE product in each case (Fig. $2 A$ ). Direct sequencing of the PCR product resulting from the $p p k 25$ mRNA shows that the initiation site is just $8 \mathrm{nt}$ upstream of the ATG (Figs. $1 \mathrm{~A}, 2 \mathrm{~A}$ ), confirming a previous report (Ben-Shahar et al., 2007). Furthermore, sequenc- ing of the PCR product resulting from the ppk25-Gal4 mRNA (Fig. $2 \mathrm{~A}$ ) indicates that transcription of this transgene initiates at the same nucleotide as the endogenous gene (Fig. $1 B$ ). In addition, RACE products for both ppk25 and ppk25-Gal4 can be detected in the legs but not in a head fraction from which the third antennal segment was removed, mirroring the distribution of full-length ppk25 mRNA detected by Northern blot (Lin et al., 2005), whereas expression of the ubiquitously expressed rp49 is detected equally in both fractions. These results show that the ppk25 and ppk25-Gal4 mRNAs initiate at the same nucleotide and are expressed in the same body parts, suggesting that expression of the ppk25-Gal4 driver reflects that of the endogenous ppk25 gene.

To further test the specificity of ppk25-Gal4 expression, and in particular its lack of expression in the brain, we used Western blots to compare levels of GFP in extracts of different body parts (Fig. $2 B$ ). Here also we find that ppk25-Gal4-driven GFP expression is readily detectable in the legs, but not in the heads (without antennae), or in the bodies of flies. In contrast, use of fru-Gal4, a driver predominantly expressed in the brain (Manoli et al., 2005; Stockinger et al., 2005), results in high levels of GFP in head extracts (Fig. $2 B$ ). Together, these data suggest that the $p p k 25$ Gal4 transgene and the endogenous ppk25 gene are regulated in similar ways, making ppk25-Gal4 a powerful tool to study the function of $p p k 25$ and the cells in which it functions.

Expression of $p p k 25-\mathrm{Gal} 4$ is restricted to small characteristic subsets of gustatory and olfactory neurons, some of which have been implicated in male courtship behavior

Drosophila gustatory neurons are located within taste hairs found mostly on the mouthparts, legs, and wings (Fig. 3A) (Shanbhag et al., 2001; Vosshall and Stocker, 2007). Under control of ppk25Gal4, mCD8-GFP expression is observed in most taste hairs of the front legs (Fig. $3 \mathrm{~B}, \mathrm{C}$ ), other legs (data not shown), and wings (Fig. 3D) of both males and females, but in no other part of the body (data not shown). Targeted expression of tau-GFP, a marker of neuronal processes in Drosophila (Murray et al., 1998) (Fig. $3 C, C^{\prime}$ ), allows visualization of a long apical dendrite through the entire hair shaft (Fig. $3 C^{\prime}$, green arrow), identifying these cells as gustatory neurons (Shanbhag et al., 2001). Interestingly, double-staining with anti-CheB42a antibody (Park et al., 2006) shows that expression of the two neighboring genes does not overlap despite the very short intergenic region (Fig. 1A); instead, $p p k 25$ expression occurs in gustatory neurons, whereas, as shown previously (Park et al., 2006), CheB42a is expressed in the surrounding non-neuronal sheath cells (Fig. $3 E, F)$. The expression of $C h e B 42 a$ and $p p k 25$ in distinct cell types and the presence of a $5^{\prime}$ cap modification on the $p p k 25$ mRNA as indicated by its amplification by RLM-RACE (Fig. $2 A$ ), together with the absence of a detectable precursor RNA containing both CheB42a and ppk25 sequences (Park et al., 2006; Ben-Shahar et al., 2007), indicate that the adjacent CheB42a and ppk25 genes are transcribed independently rather than resulting from the posttranscriptional processing of a common polycistronic transcript as previously suggested (Ben-Shahar et al., 2007).

Expression of ppk25-Gal4 defines a small subset of gustatory neurons with distinguishing characteristics. Most taste hairs on the legs contain four gustatory neurons (Shanbhag et al., 2001). Two of these taste neurons express fruitless ( fru), the master regulator of neuronal sexual determination required for most male-specific behaviors (Siwicki and Kravitz, 2009), and send characteristic sexually dimorphic projections onto the prothoracic ganglia (Mellert et al., 2010). Expression of ppk25-Gal4 is 


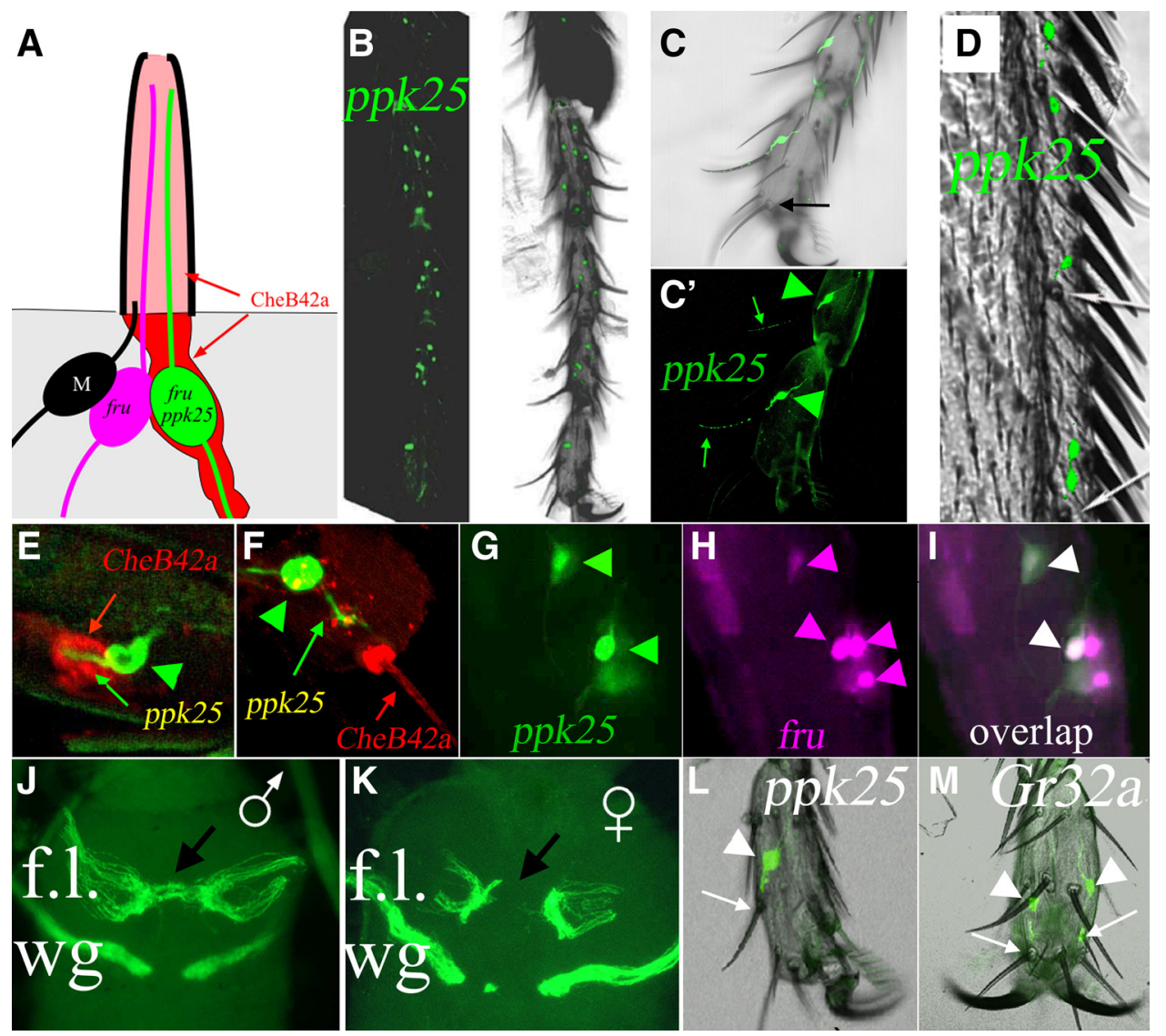

Figure 3. ppk25-Gal4 is expressed in a small subset of gustatory neurons implicated in sexual behavior. A, Schematic drawing of a gustatory hair of the leg. The dendrites of two to four gustatory neurons (only the two fru-expressing neurons are shown) extend through the hair lumen to the terminal pore where they can interact with chemicals in the environment (Shanbhag et al., 2001). One neuron expresses both ppk25 and fru (green), while another expresses fru but not ppk25 (magenta; see Results). A single mechanosensory neuron ( $M$, black) in each hair has much shorter, stout dendrites that terminate at the base of the hair (Shanbhag et al., 2001). The CheB42a protein is expressed in non-neuronal cells (red) that surround the ppk25-expressing neuron and its dendrites and is secreted into the lumen of the taste hair (Park et al., 2006; Starostina et al., 2009) (see Results). B, Expression of membrane-tethered mCD8-GFP under control of the ppk25-Gal4 driver is present in most taste hairs of all legs (male foreleg shown). $\boldsymbol{C}$, Higher magnification shows that expression is confined to only one gustatory neuron per taste hair. $\boldsymbol{C}$ and $\boldsymbol{C}^{\prime}$ show superimposed fluorescent and bright light images, or fluorescence only, respectively. In this experiment, ppk25-Gal4 drives expression of tau-GFP, a reporter protein that localizes to neurites (Murray et al., 1998). In each of two GFP-expressing cells visible in this field, a dendrite extends through the whole length of the hair shaft ( $\boldsymbol{C}^{\prime}$, green arrow), indicating that expression occurs in gustatory neurons. Of six taste hairs in the terminal leg segment (Meunier et al., 2000,2003), only two hairs in the proximal part of the segment house a mCD8-GFP-labeled gustatory neuron (only one of which is seen in this optical section). There is no reporter gene expression in taste hairs closer to the leg tip which contains food-sensing neurons (one such hair in focus in Cis indicated by a black arrow)(Meunier et al., $2000,2003)$. $\boldsymbol{D}$, In the wing also, ppk25-Gal4 drives expression of mCD8-GFP in a single gustatory neuron in each taste hair. $\boldsymbol{E}$, $\boldsymbol{F}$, CheB42a, a male-specific gene immediately upstream of $p p k 25$ (Fig. $1 \mathrm{~A}$ ) is expressed in non-neuronal support cells (Park et al., 2006; Starostina et al., 2009), in the same taste hairs as ppk25-Gal4-expressing gustatory neurons. E, CheB42a-expressing non-neuronal sheath cells labeled with anti-CheB42a antibody (red) surround a gustatory neuron that expresses mCD8-GFP under control of $p p k 25$-Gal4 (green, cell body indicated by arrowheads; dendrite by arrows). $\boldsymbol{F}$, Optical section through the shaft of a taste hair showing secreted CheB42a in the lumen of a taste hair penetrated by the dendrite of a ppk25-Gal4-expressing neuron. $\mathbf{G}-\boldsymbol{I}$, Taste neurons expressing nuclear GFP under control of ppk25-Gal 4 (G, in green) also express tdTomato::nls under control of fru-LexA (Mellert et al., 2010) $(\boldsymbol{H}, \boldsymbol{I}$, tdTomato::nls in magenta, overlap of tdTomato::nls and GFP in white). $\boldsymbol{J}, \boldsymbol{K}$, The taste neurons expressing m(D8-GFP under control of $p p k 25-G a / 4$ in the front legs send sexually dimorphic projections to the first thoracic ganglia. Gustatory neurons expressing ppk25-Gal4 in the front legs (f.I.) make projections that cross the midline in males ( $\boldsymbol{J}$, arrow), but not females $(\boldsymbol{K})$, as reported for fru-expressing gustatory neurons (Possidente and Murphey, 1989; Boll and Noll, 2002; Mellert et al., 2010). In contrast, the projections from ppk25-Gal4-expressing neurons on the wings (wg) and from the second and third pairs of legs (data not shown) do not cross the midline in flies of either sex. L, M, ppk25 and Gr32a, gustatory receptors that inhibit courtship behavior (Miyamoto and Amrein, 2008; Koganezawa et al., 2010; Wang et al., 2011), are expressed in nonoverlapping patterns. Observations of the fifth tarsal segment show that dendrites of $p p k 25$-Gal4 expressing gustatory neurons (only one of two ppk25-Gal4-expressing neurons is visible in this side view) penetrate the shaft of taste hairs in the center of the tarsal segment ( $\boldsymbol{L}$, cell body indicated by arrowhead, arrow points to base of the corresponding taste hair), whereas dendrites of gustatory neurons that express $\mathrm{Gr} 32 \mathrm{a}$-Gal4 enter taste hairs that are closer to the leg tip ( $M$, cell bodies indicated by arrowhead, arrows points to bases of the corresponding taste hairs).

restricted to a single gustatory neuron per taste hair (Fig. 3C,E-G). Identification of the ppk25-expressing cell as one of the fruexpressing neurons is demonstrated by double-labeling (Fig. $3 G-I$ ) and confirmed by the sexual dimorphism of its projections onto the prothoracic ganglia (Fig. $3 \mathrm{~J}, \mathrm{~K}$ ). Intriguingly, ppk25Gal4 is not expressed in the tips of legs, the site of several taste hairs that respond to general food components such as sugars and bitter compounds (Fig. 3C, black arrow) (Meunier et al., 2000, 2003), but is expressed in most other leg taste hairs that do not respond to food, some of which have been implicated in detection of pheromones that either stimulate or inhibit courtship behavior (Bray and Amrein, 2003; Park et al., 2006; Miyamoto and Amrein, 2008; Koganezawa et al., 2010; Lee et al., 2010; Wang et al., 2011; Watanabe et al., 2011). The specificity of $p p k 25-$ Gal4 expression is further highlighted by its absence from the many taste hairs present in other parts of the adult body (Shanbhag et al., 2001; Vosshall and Stocker, 2007; Montell, 2009). In particular, ppk25-Gal4 is not expressed in the numerous gustatory hairs found on the mouthparts (data not shown), most of which have been shown to respond to food stimuli such as sugars, salts, and bitter compounds. Finally, comparison of the expression of $p p k 25$ to that of $G r 32 a$, a gustatory receptor that inhibits male- 

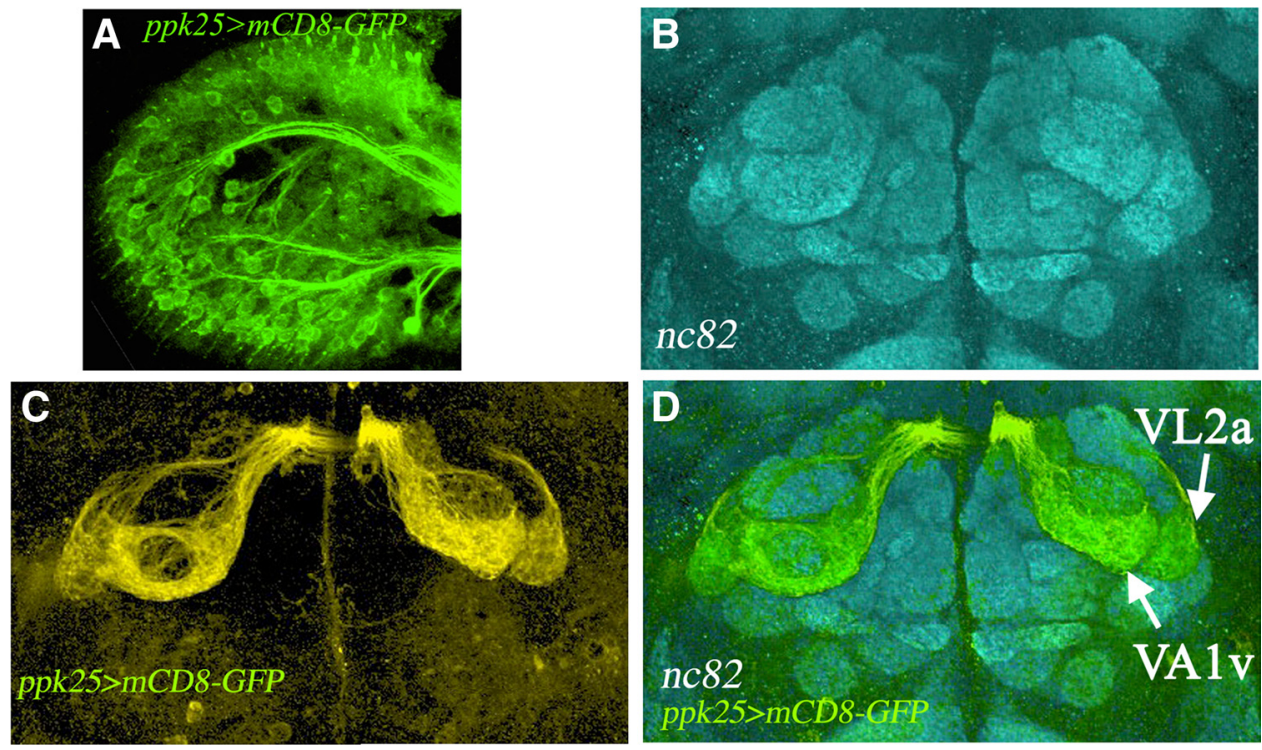

Figure 4. $\quad$ ppk25-Gal4 is expressed in two subsets of olfactory neurons that stimulate male courtship. $A$, Immunostaining reveals $m$ CD8-GFP expression in olfactory neurons within the third antennal segment of ppk25-Gal4;UAS-mCD8-GFP males. $\boldsymbol{B}$, Whole-brain immunostaining of males of the same genotype with neuronal-specific nc 82 decorates the glomeruli of the antennal lobe. C, Staining with anti-GFP reveals the axonal projections of ppk25-Gal4-expressing neurons. D, A superimposition of the two immunostaining patterns shows that the antennal neurons that express ppk25-Gal4 project to two glomeruli that are easily identified by their position on the antennal lobe and characteristic shapes: Va1v, also called Va1lm, and VL2a (Couto et al., 2005; Fishilevich and Vosshall, 2005). Both Va1v and VL2a are sexually dimorphic and express fru (Kondoh et al., 2003; Stockinger et al., 2005). VA1v receives projections from olfactory neurons that respond to female cuticular extracts and express Or47b, an olfactory receptor for fly odors (van der Goes van Naters and Carlson, 2007; Root et al., 2008; Wang et al., 2011), while VL2a is innervated by olfactory neurons that express IR84a and stimulate courtship in response to aromatic food odors (Grosjean et al., 2011).

male courtship (Miyamoto and Amrein, 2008; Koganezawa et al., 2010) in response to 7-tricosene (Wang et al., 2011), suggests that the two genes are expressed in different subsets of leg gustatory neurons (Figs. $3 L, M$ ), consistent with the absence of fruitless expression in Gr32a-expressing neurons (Miyamoto and Amrein, 2008; Koganezawa et al., 2010). While ppk25-Gal4 drives mCD8-GFP expression in all five tarsal segments on the front legs (Fig. 3C and not shown), Gr32a-Gal4-driven expression is observed only in the fourth and fifth segments ((Miyamoto and Amrein, 2008; Koganezawa et al., 2010) (data not shown). On the fifth segment, $p p k 25$-Gal4 labels two neurons that innervate hairs in the middle of the segment, whereas Gr32a-Gal4 is expressed in two neurons that innervate hairs close to the tip of that segment (3 M; Fig. $3 L$, arrowheads indicate the neuronal cell bodies; arrows point to the base of the hair penetrated by the dendrite). In the fourth tarsal segment, counts of cell bodies that express mCD8-GFP under control of ppk25-Gal4, Gr32a-Gal4, or in the presence of both transgenes consistently revealed 7,3 , and 10 taste neurons, respectively, $(n>10$ for each genotype; data not shown), suggesting that here also, expression of the two drivers does not overlap. Finally, we do not observe ppk25-Gal4-directed expression of mCD8-GFP in chemosensory neurons of the ovipositor (Stocker, 1994), and the absence of mCD8-GFP-labeled axonal projections to the abdominal ganglia (data not shown) suggests that ppk25-Gal4 is not expressed in the fru-positive internal sensory neurons of the female reproductive tract (Häsemeyer et al., 2009; Yang et al., 2009).

To determine whether ppk25-Gal4 directs lower levels of mCD8-GFP expression in other tissues, we used immunochemistry. Remarkably, the only additional reporter expression detected with this more sensitive method occurs in two subsets of antennal olfactory neurons, both of which have been implicated in olfactory stimulation of male courtship behavior (Fig. 4A). Forty-three distinct types of olfactory neurons have been described based on the expression of specific olfactory receptor (Or) genes, each projecting to a single identifiable pair of bilaterally symmetrical glomeruli in the antennal lobe (Couto et al., 2005; Fishilevich and Vosshall, 2005). ppk25-Gal4-expressing olfactory neurons project only to two pairs of glomeruli, VA1v and VL2a, identified by their shape, size, and position within the antennal lobe (Fig. $4 B-D$ ). Both VA1v and VL2a are sexually dimorphic and express fruitless (Kondoh et al., 2003; Couto et al., 2005; Fishilevich and Vosshall, 2005; Manoli et al., 2005; Stockinger et al., 2005). Furthermore, both VA1v and VL2a have been implicated in stimulating male courtship behavior, the former through detection of fly odors (van der Goes van Naters and Carlson, 2007; Root et al., 2008; Wang et al., 2011), and the latter in response to aromatic food chemicals (Grosjean et al., 2011). In summary, expression of ppk25-Gal4 is restricted to small subsets of gustatory and olfactory neurons. For both gustatory neurons of the legs and olfactory neurons of the antennae, ppk25-Gal4 expression occurs in a subset of fru-expressing neurons and includes neurons with either known or suspected roles in stimulation of male courtship behavior.

\section{A small subset of chemosensory neurons is required for male response to females}

To test whether the chemosensory neurons defined by expression of ppk25-Gal4 are important for male courtship behavior in response to females, we used targeted expression of $s h i^{t s}$, a temperature-sensitive, dominant allele of Dynamin that blocks synaptic transmission at restrictive temperatures (Kitamoto, 2001) (Fig. 5). When kept at the permissive temperature of $18^{\circ} \mathrm{C}$, males expressing $s h i^{\text {ts }}$ under control of ppk25-Gal4 and control males expressing GFP in the same pattern display similar levels of courtship as measured by the CI (the fraction of a 10 min observation period spent performing any of the steps involved in the courtship sequence $\times 100)$ (Hall, 1978). However, incubation for $1 \mathrm{~h}$ at the nonpermissive temperature of $30^{\circ} \mathrm{C}$ results in a 10 -fold lower CI for males expressing $s h i^{t s}$ relative to controls. In con- 


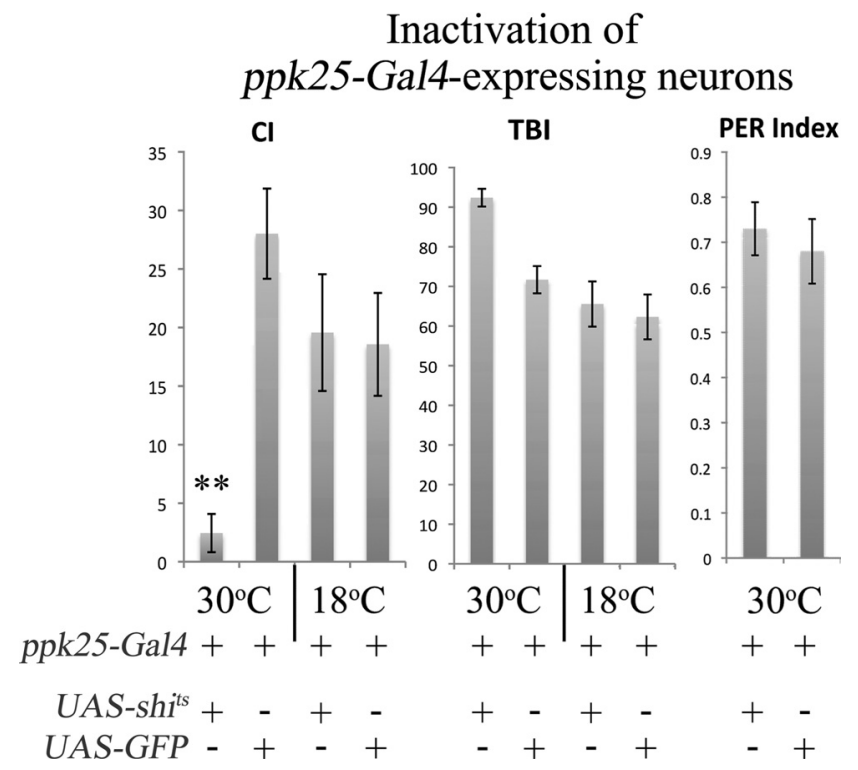

Figure 5. Inactivation of $p p k 25$-expressing neurons specifically impairs male response to virgin females. Males expressing the temperature-sensitive dominant Dynamin allele shits (Kitamoto, 2001) under control of ppk25-Gal4, were raised at the permissive temperature $\left(18^{\circ} \mathrm{C}\right)$ and, where indicated, incubated for $1 \mathrm{~h}$ at the nonpermissive temperature $\left(30^{\circ} \mathrm{C}\right)$ just before testing their behavior at the same temperature. Response to a Canton- $S$ virgin female was recorded under white light for a 10 min interval. Initiation is the fraction of males to initiate courtship within the 10 min observation period. Calculation of the $\mathrm{Cl}$, the TBI, the PER, and the corresponding SEs and statistical tests are described in Materials and Methods. ${ }^{* *} p<0.01$. For both genotypes, $N=28$ and $N=22$ at $30^{\circ} \mathrm{C}$ and $18^{\circ} \mathrm{C}$, respectively.

trast, $s h i^{t s}$-mediated inactivation of $p p k 25$-Gal4-expressing neurons does not affect the TBI (fraction of time the test male spends performing any of the most common behaviors: courtship, walking, or preening, multiplied by 100), or gustatory response to sucrose as measured by the PER (Fig. 5, right) (Heimbeck et al., 2001). These results indicate that the peripheral chemosensory neurons that express ppk25-Gal4 play an important and specific role in stimulating male courtship behavior in response to females.

ppk25 function in a small subset of gustatory neurons is required and sufficient for normal male courtship behavior in response to females

The similarity of the phenotypes caused by ppk25-Gal4-targeted expression of $s h i^{t s}$ and by mutations in ppk25 (Lin et al., 2005) suggests that $p p k 25$ functions in the peripheral chemosensory neurons in which ppk25-Gal4 is expressed. Indeed, ppk25-Gal4directed expression of wild-type $p p k 25$, but not of a mutant truncated $p p k 25\left(p p k 25^{\Delta}\right)$, is sufficient to restore a normal CI to ppk25 mutant males (Fig. 6A), but does not affect other behaviors (Fig. $6 B$ ), consistent with a specific function for $p p k 25$ in courtship behavior (Lin et al., 2005). In contrast, expression of $p p k 25$ in olfactory neurons known to respond to a small number of food odors using the Or22a-Gal4 driver (Hallem et al., 2004) has no effect on male courtship (Fig. 6A). These results suggest that the endogenous $p p k 25$ gene functions in the peripheral chemosensory neurons that express $p p k 25-G a l 4$ and raise the question of whether this function occurs in gustatory neurons, in olfactory neurons, or in both types of sensory neurons. Interestingly, while ppk25-Gal4 is expressed in olfactory neurons that project to the VA1v glomeruli which have been implicated in response to fly odors (van der Goes van Naters and Carlson, 2007; Root et al., 2008; Wang et al., 2011), targeted expression of $p p k 25$ in neurons

\section{Targeted Rescue of ppk25 mutations}

A

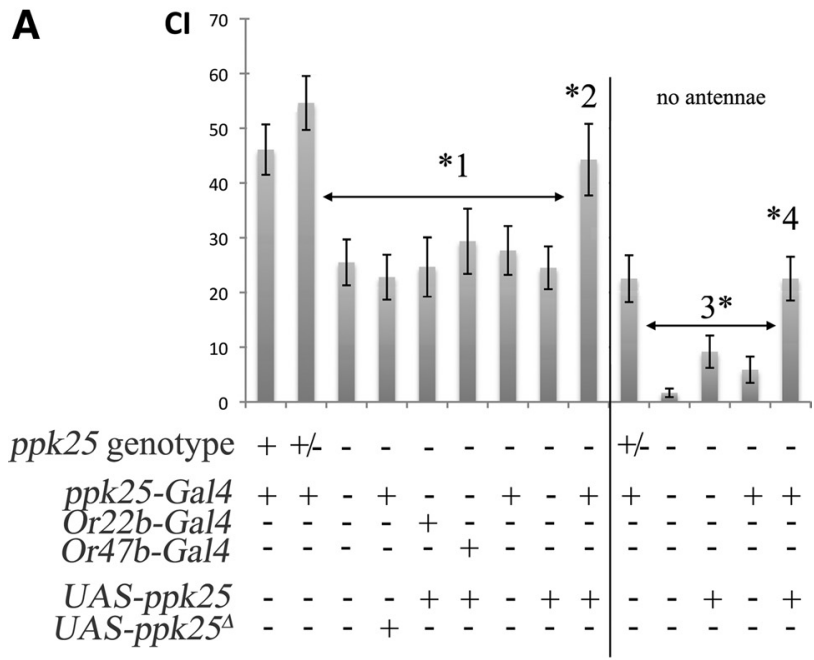

B

TBI 100

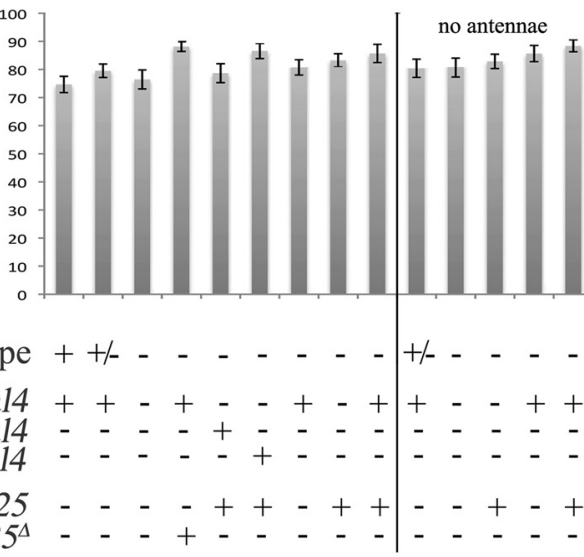

C

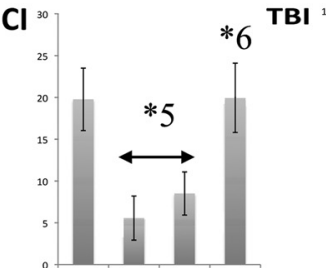

ppk25 genotype $+-\quad-\quad-$

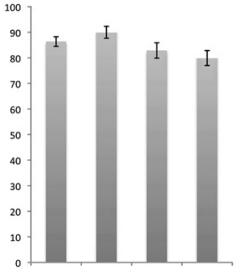

Poxn-Gal4 + + + +

$+$

UAS-ppk25 - - - +

UAS-ppk $25^{-}$

Figure 6. Targeted expression of $p p k 25$ in gustatory neurons rescues the courtship behavior of ppk25 mutant males. Courtship behavior was measured for the males containing the transgenes indicated, and of the following genotypes: control males that are wild-type for ppk25, +; males heterozygous for a deletion spanning $p p k 25,+1-$; males heterozygous for two different deletions spanning the ppk25 gene, - . A, targeted expression of ppk25 using ppk25-Gal 4 rescues male courtship. Right panel, labeled as "no antennae," shows experiments in which the third antennal segment was removed bilaterally. In all cases, ${ }^{*}$ indicates a statistically significant difference, $p<0.05$. Statistically significant differences are as follows: ${ }^{*} 1$, between control males carrying one or two copies of the wild-type ppk25 gene and ppk25 mutants with various combinations of transgenes; and *2, between homozygous ppk25 mutants expressing ppk25 under control of ppk25-Gal4 and homozygous ppk25 mutants carrying other combinations of transgenes. ${ }^{*},{ }^{*} 4$ refer to the corresponding differences among antennaless males. $\boldsymbol{B}$, Males of all genotypes have a similar TBI. $\boldsymbol{C}$, The ppk25 mutant phenotype is rescued by targeted expression of $p p k 25$ in all gustatory neurons using the Poxn-Gal4 driver (Boll and Noll, 2002). Statistically significant differences between control males and ppk25 mutant males with various transgenes, and between ppk25 mutants with various transgenes and males expressing $p p k 25$ under control of Poxn-Gal4, are indicated by ${ }^{*} 5$ and ${ }^{*} 6$, respectively. Here also, males of all genotypes have similar TBls. 


\section{Targeted RNAi}

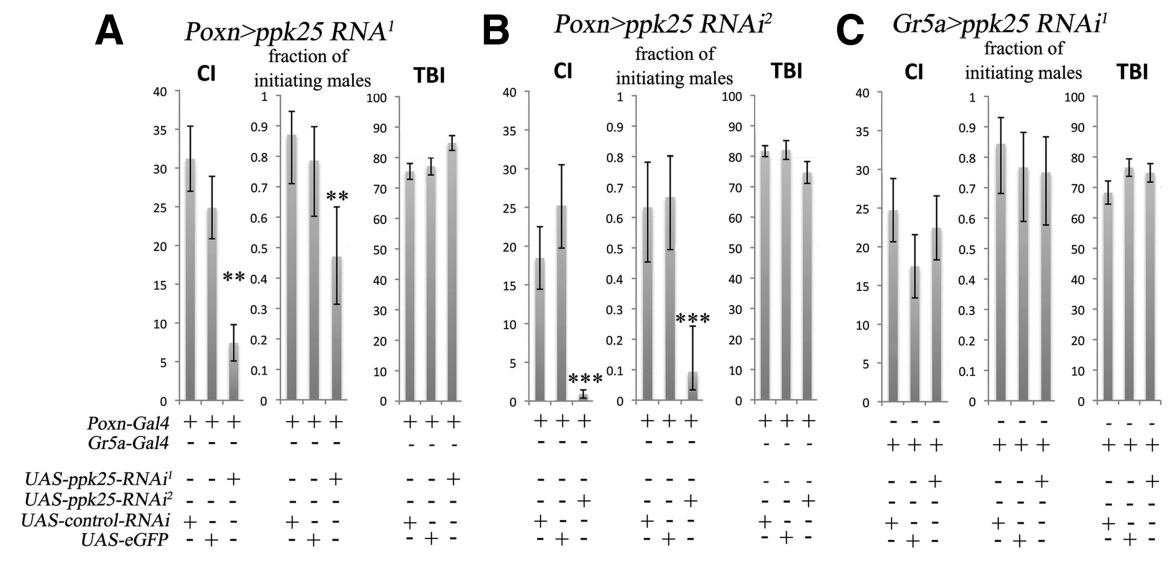

Figure 7. Targeted RNAi knockdown of $p p k 25$ in gustatory neurons blocks male courtship behavior. $A$, Gustatory expression of $p p k 25$ RNAi ${ }^{1}$ using the Poxn-Gal 4 driver (Boll and Noll, 2002 ) reduces both $\mathrm{Cl}$ and the fraction of initiating males. Control males expressed either eGFP, or a control RNAi against CG13895, a gene with no known involvement in mating behavior (Benchabane et al., 2011), in the same pattern. ${ }^{* *} p<0.01$. $B$, Gustatory expression of a second RNAi transgene, $p p k 25 R N A i^{2}$, using the Poxn-Gal4 driver reduces both $\mathrm{Cl}$ and the fraction of initiating males. This experiment was performed at $27^{\circ} \mathrm{C}$ because preliminary tests at $25^{\circ} \mathrm{C}$ resulted in effects that were not statistically significant at $p<0.05$ (data not shown). ${ }^{* *} p<0.05$; ${ }^{* * *} p<0.001$. C, As in $A$, except for the use of $\mathrm{Gr} 5 a-G a / 4$ to drive expression in sucrose-sensing neurons of the labelum and legs (Chyb et al., 2003). Expression of $p p k 25 R N A i^{1}$ in those neurons does not result in any significant effect on male courtship.

projecting to the same glomeruli using the Or47b-Gal4 driver does not rescue the courtship of $p p k 25$ mutant males. To evaluate more directly the importance of the antennal expression of $p p k 25$, we tested whether removing the main olfactory organ, the third antennal segment (Masse et al., 2009), affected ppk25-Gal4directed rescue of $p p k 25$ mutants. Consistent with other reports, loss of olfaction reduces male response to females by approximately 2-fold (Stockinger et al., 2005; Krstic et al., 2009) (Fig. $6 A)$. More important, courtship of such antennaless males is further reduced in mutants lacking the $p p k 25$ gene and targeted expression of ppk25 using ppk25-Gal4 rescues their response to females to the level displayed by antennaless wild-type males, whereas the presence of either parental transgene alone has no significant effect (Fig. 6A). In contrast, neither removal of the antennae nor expression of functional $p p k 25$ affects the total behavioral output of males (Fig. 6B). These data suggest that in the absence of antennae at least, expression of $p p k 25$ in the small subset of ppk25-Gal4-expressing gustatory neurons on the legs and wings is sufficient for normal male courtship.

To further test whether $p p k 25$ functions in gustatory neurons, we asked whether targeted expression of ppk25 using Poxn-Gal4, a driver expressed specifically and at high levels in all gustatory neurons (Boll and Noll, 2002), was sufficient to rescue the behavioral phenotype of $p p k 25$ mutant males (Fig. $6 A$ ). In preliminary experiments, we were surprised to find that in $p p k 25$ heterozygotes, the Poxn-Gal4 driver itself inhibits male courtship behavior by $\sim 2$-fold (Fig. 6, compare $A$ and $C$ and data not shown), perhaps as a result of high levels of the Gal4 protein in gustatory neurons, as reported for other subsets of gustatory neurons (Lacaille et al., 2007; Yang et al., 2008), and other types of neurons (Rezával et al., 2007). Nevertheless, comparison of a series of males that all carry the Poxn-Gal4 driver shows that inactivation of the $p p k 25$ gene results in a significant further reduction of male response to females, and that Poxn-Gal4-directed expression of $p p k 25$, but not of a truncated $p p k 25^{\Delta}$, rescues male behavior to the level found in the presence of the endogenous ppk25 (Fig. 6C). Here also, males of all genotypes have similar TBIs (Fig. 6C). These experiments further support the conclusion that gustatory expression of $p p k 25$ is sufficient for normal male courtship.
In complementary experiments, we tested the effect on male courtship of knocking down the ppk25 mRNA specifically in gustatory neurons using two different UAS-ppk25 RNAi transgenes (Dietzl et al., 2007; Ni et al., 2009) under control of Poxn-Gal4. Males expressing either ppk25 RNAi under control of Poxn-Gal4 display severely reduced levels of overall courtship but normal levels of total behavior compared with control males expressing an unrelated $R N A i$ or a GFP reporter in the same neurons (Fig. $7 A, B)$. In contrast, expression of $p p k 25 R N A i$ in sugar-sensing gustatory neurons using the Gr5a-Gal4 driver (Chyb et al., 2003) does not affect courtship behavior (Fig. 7C) or response to sucrose as measured by PER (data not shown). Furthermore, the effect of gustatory-specific knockdown of ppk25 is not restricted to late steps in courtship behavior, as the fraction of males that initiate courtship is also severely reduced relative to controls (Fig. 7; $p<0.01$ and $p<0.001$ for $p p k 25-R N A i^{1}$ and $p p k 25-R N A i^{2}$, respectively). These results indicate that $p p k 25$ function in gustatory neurons is required for normal courtship, starting from the earliest steps in this behavioral sequence.

In summary, our data reveal that under our experimental conditions ppk25 function in a small and specific subset of gustatory neurons on the legs and wings is both necessary and sufficient for normal activation of male courtship behavior in response to females.

\section{Discussion}

\section{Identification of a subset of gustatory neurons required for} activation of male courtship of females

Activation of the courtship behavior of Drosophila melanogaster males involves detection of specific female pheromones, including several well characterized hydrocarbons (Antony et al., 1985; Ferveur et al., 1997; Ferveur, 2005; Siwicki et al., 2005; Billeter et al., 2009). While the sense of smell plays a role in courtship activation (Villella and Hall, 2008), gustatory detection appears to be more important (Fig. 7) (Boll and Noll, 2002; Krstic et al., 2009). However, before this work, the gustatory neurons involved in detecting courtship-activating pheromones remained uncertain. Here we identify a small subset of gustatory neurons on the legs and wings of males that have an essential function in the activa- 
tion of courtship behavior. Use of a ppk25-Gal4 transgene to specifically inhibit synaptic function in small subsets of gustatory and olfactory neurons dramatically reduces male courtship of females, while expression of $p p k 25$ in the same neurons rescues the defective courtship of ppk25 mutant males. Additional experiments further localize this function to the gustatory neurons on the legs and wings where $p p k 25-G a l 4$ is expressed. First, the $p p k 25$ mutant phenotype is rescued by gustatory-specific expression of ppk25 by two different methods: (1) using ppk25-Gal4 to drive ppk25 expression in males lacking their antennae, the site of the only detectable expression of ppk25-Gal4 outside of the gustatory system, and (2) using the gustatory-specific Poxn-Gal4 driver. Second, gustatory-specific knockdown of ppk25 function using two distinct $p p k 25$ RNAis under control of Poxn-Gal4 reduces the levels of male courtship displayed by factors of 7 and 20. Together, these results indicate that expression of $p p k 25$ in the small subsets of gustatory neurons defined by expression of $p p k 25-G a l 4$ on the legs and wings of males are both required and under laboratory conditions, at least, are sufficient for normal activation of male courtship behavior.

These findings have implications not only for the gustatory function of $p p k 25$, but also for its possible involvement in olfaction. The very low levels of courtship displayed by males after gustatory-specific RNAi-mediated knockdown of ppk25 suggest that expression of $p p k 25$ in olfactory neurons is not sufficient to initiate courtship. Furthermore, since olfaction allows detection of volatile chemicals at a distance from their source, it has been generally assumed that olfactory detection is solely responsible for the effects of pheromones on the initiation of male courtship, while gustatory perception of pheromones becomes possible only after direct contact of the gustatory hairs of the male with the female body. The severe defects displayed by males carrying different $p p k 25$ alleles in both early and late events in courtship therefore led us to speculate that $p p k 25$ may function in both chemical senses (Lin et al., 2005). However, we find that specific knockdown of ppk25 mRNA in taste neurons significantly reduces the fraction of males that initiate any courtship behavior (Fig. $7 A, B$ ). These data confirm a previous report that gustatory perception plays a key role not only in the maintenance, but also in the initiation of courtship behavior (Krstic et al., 2009). Gustatory stimulation may occur as the male and female touch by chance before any courtship behavior has been displayed, or alternatively as the male comes in direct contact with female pheromones deposited on the surface of the observation chamber in a mechanism analogous to the contact detection of low volatility trail pheromones in some ants and caterpillars (Roessingh et al., 1988; Jackson et al., 2006). These data indicate that $p p k 25$ functions in gustatory neurons that have an important role, not only in the late steps of male courtship, but also in the initiation of this complex behavior.

It is nonetheless remarkable that the only detectable expression of ppk25-Gal4 outside of the gustatory system, albeit at a significantly lower level, occurs in two subsets of sexually dimorphic olfactory neurons, both of which express fruitless and stimulate courtship behavior either in direct response to female odors (van der Goes van Naters and Carlson, 2007; Wang et al., 2011), or through detection of aromatic fruit compounds that enhance the attractiveness of females (Grosjean et al., 2011). ppk25 may therefore have a role in olfactory modulation of courtship that would only become apparent under conditions that enhance the importance of olfactory inputs (Ejima and Griffith, 2008; Root et al., 2008; Grosjean et al., 2011).
What are the ligand(s) detected by the gustatory neurons that express ppk25-Gal4? Synaptic inactivation of those neurons does not affect gustatory response to sugar (Fig. 5), or result in increased male-male courtship as seen in males deficient in detection of courtship-inhibiting pheromones (data not shown) (Kurtovic et al., 2007; Miyamoto and Amrein, 2008). In addition, expression of ppk25-Gal4 does not occur on the proboscis or leg tips where many specialized food-sensing hairs are located. Finally, expression of ppk25-Gal4 in the taste hairs of the legs only occurs in one of the two fru-positive neurons present, and does not overlap with that of Gr32a, a taste receptor that inhibits male courtship behavior (Miyamoto and Amrein, 2008) through detection of 7-tricosene (Wang et al., 2011). Together, these data suggest that the gustatory neurons defined by expression of ppk25-Gal4 have a specialized role in detecting one or more ligands that activate courtship behavior. The relevant ligands remain to be identified, and it will be of particular interest to test whether they include one or more of the known low-volatility female pheromones that activate male courtship (Antony et al., 1985; Ferveur et al., 1997; Ferveur, 2005; Siwicki et al., 2005; Billeter et al., 2009).

\section{A DEG/ENaC channel with a specific role in gustatory activation of courtship behavior}

What is the molecular mechanism that underlies $p p k 25$ 's role in gustatory activation of male courtship? ppk25 may have a direct role in gustatory detection of a courtship-stimulating molecule, either as part of a receptor complex, or further downstream in the signaling cascade. While such mechanisms deviate from the well known G-protein-coupled processes involved in vertebrate chemical senses (Pifferi et al., 2010), recent findings suggest that in insects, both olfaction and taste function through ion channels that are directly gated by environmental ligands and belong to multiple structurally distinct families (Silbering and Benton, 2010). In particular, another Drosophila DEG/ENaC subunit, $p p k 28$, mediates gustatory response to water and its ectopic expression confers osmotic sensitivity to nonresponsive neurons, suggesting that gating of $p p k 28$ must be controlled by osmotic pressure (Cameron et al., 2010; Chen et al., 2010). In vertebrates, $\mathrm{ENaC}$ expressed in taste cells functions as a salt receptor, depolarization resulting directly from the specific translocation of extracellular sodium (Schiffman et al., 1983; Heck et al., 1984; Chandrashekar et al., 2010), and similar mechanisms may be involved for two Drosophila DEG/ENaCs implicated in salt response, $p$ pk11 and ppk19 (Liu et al., 2003). Finally, ligand-gated $\mathrm{DEG} / \mathrm{ENaCs}$ are also found outside of the gustatory system, e.g., protons and other ligands trigger opening of the acid-sensing channel (Yu et al., 2010), and FRMF-amide gates the gastropod FRMF-amide receptor (Poët et al., 2001). Gating of ppk25 may therefore be controlled by courtship-activating molecules, either directly or in a complex with pheromone-binding proteins in a mechanism related to olfactory detection of cis-vaccenyl acetate, an inhibitory volatile pheromone (Laughlin et al., 2008). An alternative possibility is that $p p k 25$ may play a less direct role in modulating the response of courtship-activating gustatory neurons. By analogy to a Caenorhabditis elegans DEG/ENaC channel that mediates learning through presynaptic facilitation of dopamine release (Voglis and Tavernarakis, 2008), ppk25 may exert its effect on neuronal function in the axon or at the synaptic terminal rather than in sensory dendrites. This work opens the way to the functional characterization of gustatory activation of male courtship, and in particular to the dissection of the molecular role 
of ppk25 and other DEG/ENaC channel subunits in gustatory detection of pheromones and other organic molecules.

\section{References}

Antony C, Davis TL, Carlson DA, Pechine JM, Jallon JM (1985) Compared behavioral responses of males Drosophila melanogaster (Canton-S) to natural and synthetic aphrodisiacs. J Chem Ecol 11:1617-1629.

Benchabane H, Xin N, Tian A, Hafler BP, Nguyen K, Ahmed A, Ahmed Y (2011) Jerky/Earthbound facilitates cell-specific Wnt/Wingless signalling by modulating beta-catenin-TCF activity. EMBO J 30:1444-1458.

Ben-Shahar Y, Nannapaneni K, Casavant TL, Scheetz TE, Welsh MJ (2007) Eukaryotic operon-like transcription of functionally related genes in Drosophila. Proc Natl Acad Sci U S A 104:222-227.

Billeter JC, Atallah J, Krupp JJ, Millar JG, Levine JD (2009) Specialized cells tag sexual and species identity in Drosophila melanogaster. Nature 461:987-991.

Boll W, Noll M (2002) The Drosophila Pox neuro gene: control of male courtship behavior and fertility as revealed by a complete dissection of all enhancers. Development 129:5667-5681.

Brand AH, Dormand EL (1995) The GAL4 system as a tool for unravelling the mysteries of the Drosophila nervous system. Curr Opin Neurobiol 5:572-578.

Bray S, Amrein H (2003) A putative Drosophila pheromone receptor expressed in male-specific taste neurons is required for efficient courtship. Neuron 39:1019-1029.

Cameron P, Hiroi M, Ngai J, Scott K (2010) The molecular basis for water taste in Drosophila. Nature 465:91-95.

Chandrashekar J, Kuhn C, Oka Y, Yarmolinsky DA, Hummler E, Ryba NJ, Zuker CS (2010) The cells and peripheral representation of sodium taste in mice. Nature 464:297-301.

Chen Z, Wang Q, Wang Z (2010) The amiloride-sensitive epithelial $\mathrm{Na}+$ channel PPK28 is essential for Drosophila gustatory water reception. J Neurosci 30:6247-6252.

Chyb S, Dahanukar A, Wickens A, Carlson JR (2003) Drosophila Gr5a encodes a taste receptor tuned to trehalose. Proc Natl Acad Sci U S A 100 [Suppl] 2:14526-14530.

Couto A, Alenius M, Dickson BJ (2005) Molecular, anatomical, and functional organization of the Drosophila olfactory system. Curr Biol 15:1535-1547.

Dahanukar A, Ray A (2011) Courtship, aggression and avoidance: pheromones, receptors and neurons for social behaviors in Drosophila. Fly 5:58-63.

Datta SR, Vasconcelos ML, Ruta V, Luo S, Wong A, Demir E, Flores J, Balonze K, Dickson BJ, Axel R (2008) The Drosophila pheromone cVA activates a sexually dimorphic neural circuit. Nature 452:473-477.

Dietzl G, Chen D, Schnorrer F, Su KC, Barinova Y, Fellner M, Gasser B, Kinsey K, Oppel S, Scheiblauer S, Couto A, Marra V, Keleman K, Dickson BJ (2007) A genome-wide transgenic RNAi library for conditional gene inactivation in Drosophila. Nature 448:151-156.

Ejima A, Griffith LC (2008) Courtship initiation is stimulated by acoustic signals in Drosophila melanogaster. PLoS ONE 3:e3246.

Elliott DA, Brand AH (2008) The GAL4 system: a versatile system for the expression of genes. Methods Mol Biol 420:79-95.

Ferveur JF (2005) Cuticular hydrocarbons: their evolution and roles in Drosophila pheromonal communication. Behav Genet 35:279-295.

Ferveur JF, Savarit F, O'Kane CJ, Sureau G, Greenspan RJ, Jallon JM (1997) Genetic feminization of pheromones and its behavioral consequences in Drosophila males. Science 276:1555-1558.

Fishilevich E, Vosshall LB (2005) Genetic and functional subdivision of the Drosophila antennal lobe. Curr Biol 15:1548-1553.

Griffith LC, Ejima A (2009) Multimodal sensory integration of courtship stimulating cues in Drosophila melanogaster. Ann NY Acad Sci 1170:394-398.

Grosjean Y, Rytz R, Farine JP, Abuin L, Cortot J, Jefferis GS, Benton R (2011) An olfactory receptor for food-derived odours promotes male courtship in Drosophila. Nature 478:236-240.

Hall JC (1978) Courtship among males due to a male-sterile mutation in Drosophila melanogaster. Behav Genet 8:125-141.

Hallem EA, Ho MG, Carlson JR (2004) The molecular basis of odor coding in the Drosophila antenna. Cell 117:965-979.

Häsemeyer M, Yapici N, Heberlein U, Dickson BJ (2009) Sensory neurons in the Drosophila genital tract regulate female reproductive behavior. Neuron 61:511-518.

Heck GL, Mierson S, DeSimone JA (1984) Salt taste transduction occurs through an amiloride-sensitive sodium transport pathway. Science 223:403-405.

Heimbeck G, Bugnon V, Gendre N, Keller A, Stocker RF (2001) A central neural circuit for experience-independent olfactory and courtship behavior in Drosophila melanogaster. Proc Natl Acad Sci U S A 98:15336-15341.

Jackson DE, Martin SJ, Holcombe M, Ratnieks FLW (2006) Longevity and detection of persistent foraging trails in Pharaoh's ants, Monomorium pharaonis. Anim Behav 71:351-359.

Kitamoto T (2001) Conditional modification of behavior in Drosophila by targeted expression of a temperature-sensitive shibire allele in defined neurons. J Neurobiol 47:81-92.

Koganezawa M, Haba D, Matsuo T, Yamamoto D (2010) The shaping of male courtship posture by lateralized gustatory inputs to male-specific interneurons. Curr Biol 20:1-8.

Kondoh Y, Kaneshiro KY, Kimura K, Yamamoto D (2003) Evolution of sexual dimorphism in the olfactory brain of Hawaiian Drosophila. Proc Biol Sci 270:1005-1013.

Krstic D, Boll W, Noll M (2009) Sensory integration regulating male courtship behavior in Drosophila. PLoS ONE 4:e4457.

Kurtovic A, Widmer A, Dickson BJ (2007) A single class of olfactory neurons mediates behavioural responses to a Drosophila sex pheromone. Nature 446:542-546.

Lacaille F, Hiroi M, Twele R, Inoshita T, Umemoto D, Manière G, MarionPoll F, Ozaki M, Francke W, Cobb M, Everaerts C, Tanimura T, Ferveur JF (2007) An inhibitory sex pheromone tastes bitter for Drosophila males. PLoS ONE 2:e661.

Laissue PP, Reiter C, Hiesinger PR, Halter S, Fischbach KF, Stocker RF (1999) Three-dimensional reconstruction of the antennal lobe in Drosophila melanogaster. J Comp Neurol 405:543-552.

Laughlin JD, Ha TS, Jones DN, Smith DP (2008) Activation of pheromone-sensitive neurons is mediated by conformational activation of pheromone-binding protein. Cell 133:1255-1265.

Lee Y, Kim SH, Montell C (2010) Avoiding DEET through insect gustatory receptors. Neuron 67:555-561.

Lin H, Mann KJ, Starostina E, Kinser RD, Pikielny CW (2005) A Drosophila $\mathrm{deg} / \mathrm{ENaC}$ channel subunit is required for male response to female pheromones. Proc Natl Acad Sci U S A 102:12831-12836.

Liu L, Leonard AS, Motto DG, Feller MA, Price MP, Johnson WA, Welsh MJ (2003) Contribution of Drosophila DEG/ENaC genes to salt taste. Neuron 39:133-146.

Manoli DS, Foss M, Villella A, Taylor BJ, Hall JC, Baker BS (2005) Malespecific fruitless specifies the neural substrates of Drosophila courtship behaviour. Nature 436:395-400.

Masse NY, Turner GC, Jefferis GS (2009) Olfactory information processing in Drosophila. Curr Biol 19:R700-R713.

Mellert DJ, Knapp JM, Manoli DS, Meissner GW, Baker BS (2010) Midline crossing by gustatory receptor neuron axons is regulated by fruitless, doublesex and the Roundabout receptors. Development 137:323-332.

Meunier N, Ferveur JF, Marion-Poll F (2000) Sex-specific non-pheromonal taste receptors in Drosophila. Curr Biol 10:1583-1586.

Meunier N, Marion-Poll F, Rospars JP, Tanimura T (2003) Peripheral coding of bitter taste in Drosophila. J Neurobiol 56:139-152.

Miyamoto T, Amrein H (2008) Suppression of male courtship by a Drosophila pheromone receptor. Nat Neurosci 11:874-876.

Montell C (2009) A taste of the Drosophila gustatory receptors. Curr Opin Neurobiol 19:345-353.

Murray MJ, Merritt DJ, Brand AH, Whitington PM (1998) In vivo dynamics of axon pathfinding in the Drosophilia CNS: a time-lapse study of an identified motorneuron. J Neurobiol 37:607-621.

Ni JQ, Liu LP, Binari R, Hardy R, Shim HS, Cavallaro A, Booker M, Pfeiffer BD, Markstein M, Wang H, Villalta C, Laverty TR, Perkins LA, Perrimon N (2009) A Drosophila resource of transgenic RNAi lines for neurogenetics. Genetics 182:1089-1100.

Park SK, Mann KJ, Lin H, Starostina E, Kolski-Andreaco A, Pikielny CW (2006) A Drosophila protein specific to pheromone-sensing gustatory hairs delays males' copulation attempts. Curr Biol 16:1154-1159.

Pfeiffer BD, Jenett A, Hammonds AS, Ngo TT, Misra S, Murphy C, Scully A, Carlson JW, Wan KH, Laverty TR, Mungall C, Svirskas R, Kadonaga 
JT, Doe CQ, Eisen MB, Celniker SE, Rubin GM (2008) Tools for neuroanatomy and neurogenetics in Drosophila. Proc Natl Acad Sci U S A 105:9715-9720.

Pifferi S, Menini A, Kurahashi T (2010) Signal transduction in vertebrate olfactory cilia. In: The neurobiology of olfaction (Menini A, ed). Boca Raton, FL: CRC.

Poët M, Tauc M, Lingueglia E, Cance P, Poujeol P, Lazdunski M, Counillon L (2001) Exploration of the pore structure of a peptide-gated $\mathrm{Na}+$ channel. EMBO J 20:5595-5602.

Possidente DR, Murphey RK (1989) Genetic control of sexually dimorphic axon morphology in Drosophila sensory neurons. Dev Biol 132:448-457.

Qian S, Capovilla M, Pirrotta V (1991) The bx region enhancer, a distant cis-control element of the Drosophila Ubx gene and its regulation by hunchback and other segmentation genes. EMBO J 10:1415-1425.

Rezával C, Werbajh S, Ceriani MF (2007) Neuronal death in Drosophila triggered by GAL4 accumulation. Eur J Neurosci 25:683-694.

Roessingh P, Peterson SC, Fitzgerald TD (1988) The sensory basis of trail following in some lepidopterous larvae: contact chemoreception. Physiol Entomol 13:219-224.

Ronderos DS, Smith DP (2010) Activation of the T1 neuronal circuit is necessary and sufficient to induce sexually dimorphic mating behavior in Drosophila melanogaster. J Neurosci 30:2595-2599.

Root CM, Masuyama K, Green DS, Enell LE, Nässel DR, Lee CH, Wang JW (2008) A presynaptic gain control mechanism fine-tunes olfactory behavior. Neuron 59:311-321.

Rubin GM, Spradling AC (1982) Genetic transformation of Drosophila with transposable elements. Science 218:348-353.

Ruta V, Datta SR, Vasconcelos ML, Freeland J, Looger LL, Axel R (2010) A dimorphic pheromone circuit in Drosophila from sensory input to descending output. Nature 468:686-690.

Schaefer BC (1995) Revolutions in rapid amplification of cDNA ends: new strategies for polymerase chain reaction cloning of full-length cDNA ends. Anal Biochem 227:255-273.

Schiffman SS, Lockhead E, Maes FW (1983) Amiloride reduces the taste intensity of $\mathrm{Na}+$ and $\mathrm{Li}+$ salts and sweeteners. Proc Natl Acad Sci U S A 80:6136-6140.

Shanbhag SR, Park SK, Pikielny CW, Steinbrecht RA (2001) Gustatory organs of Drosophila melanogaster: fine structure and expression of the putative odorant-binding protein PBPRP2. Cell Tissue Res 304:423-437.

Silbering AF, Benton R (2010) Ionotropic and metabotropic mechanisms in chemoreception: 'chance or design'? EMBO Rep 11:173-179.

Siwicki KK, Kravitz EA (2009) Fruitless, doublesex and the genetics of social behavior in Drosophila melanogaster. Curr Opin Neurobiol 19:200-206.

Siwicki KK, Riccio P, Ladewski L, Marcillac F, Dartevelle L, Cross SA, Ferveur
JF (2005) The role of cuticular pheromones in courtship conditioning of Drosophila males. Learn Mem 12:636-645.

Starostina E, Xu A, Lin H, Pikielny CW (2009) A Drosophila protein family implicated in pheromone perception is related to Tay-Sachs GM2activator protein. J Biol Chem 284:585-594.

Stocker RF (1994) The organization of the chemosensory system in Drosophila melanogaster: a review. Cell Tissue Res 275:3-26.

Stockinger P, Kvitsiani D, Rotkopf S, Tirián L, Dickson BJ (2005) Neural circuitry that governs Drosophila male courtship behavior. Cell 121:795-807

van der Goes van Naters W, Carlson JR (2007) Receptors and neurons for fly odors in Drosophila. Curr Biol 17:606-612.

Villella A, Hall JC (2008) Neurogenetics of courtship and mating in Drosophila. Adv Genet 62:67-184.

Villella A, Ferri SL, Krystal JD, Hall JC (2005) Functional analysis of fruitless gene expression by transgenic manipulations of Drosophila courtship. Proc Natl Acad Sci U S A 102:16550-16557.

Voglis G, Tavernarakis N (2008) A synaptic DEG/ENaC ion channel mediates learning in $\mathrm{C}$. elegans by facilitating dopamine signalling. EMBO J 27:3288-3299.

Vosshall LB, Stocker RF (2007) Molecular architecture of smell and taste in Drosophila. Annu Rev Neurosci 30:505-533.

Wang L, Han X, Mehren J, Hiroi M, Billeter JC, Miyamoto T, Amrein H, Levine JD, Anderson DJ (2011) Hierarchical chemosensory regulation of male-male social interactions in Drosophila. Nat Neurosci 14:757-762.

Watanabe K, Toba G, Koganezawa M, Yamamoto D (2011) Gr39a, a highly diversified gustatory receptor in Drosophila, has a role in sexual behavior. Behav Genet 41:746-753

Wicker-Thomas C (2007) Pheromonal communication involved in courtship behavior in Diptera. J Insect Physiol 53:1089-1100.

Xu A, Park SK, D’Mello S, Kim E, Wang Q, Pikielny CW (2002) Novel genes expressed in subsets of chemosensory sensilla on the front legs of male Drosophila melanogaster. Cell Tissue Res 307:381-392.

Yang CH, Belawat P, Hafen E, Jan LY, Jan YN (2008) Drosophila egg-laying site selection as a system to study simple decision-making processes. Science 319:1679-1683.

Yang CH, Rumpf S, Xiang Y, Gordon MD, Song W, Jan LY, Jan YN (2009) Control of the postmating behavioral switch in Drosophila females by internal sensory neurons. Neuron 61:519-526.

Yew JY, Dreisewerd K, Luftmann H, Müthing J, Pohlentz G, Kravitz EA (2009) A new male sex pheromone and novel cuticular cues for chemical communication in Drosophila. Curr Biol 19:1245-1254.

Yu Y, Chen Z, Li WG, Cao H, Feng EG, Yu F, Liu H, Jiang H, Xu TL (2010) A nonproton ligand sensor in the acid-sensing ion channel. Neuron 68:61-72. 